\section{Numérique et santé (9) \\ Opportunités \\ et enjeux \\ de la technologie \\ blockchain dans le \\ secteur de la santé}

Anca Petre, Nassima Haï

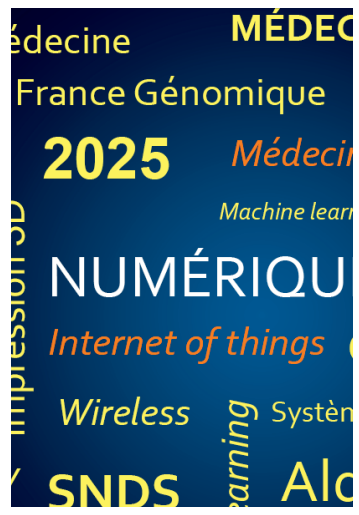

23 Consulting, 2, rue Edmond

About, 92350 Le Plessis

Robinson, France.

ancapetre.pro@gmail.com

à l'heure actuelle. Le caractère centralisé de ces supports de stockage permet de les cibler plus facilement, ce qui compromet les données qu'ils contiennent.

La blockchain, technologie de gestion décentralisée des informations, pourrait renforcer la sécurité des données sensibles. Cette technologie se matérialise par un logiciel libre (open source) qui enregistre toutes les transactions effectuées dans un réseau. Les membres de ce réseau peuvent prendre deux rôles : simples utilisateurs (ils réalisent des transactions) ou mineurs. Ces derniers s'assurent de la validité des informations transmises entre plusieurs entités et veillent au bon fonctionnement du réseau. Initialement, cette technologie a été utilisée dans le secteur financier pour tenir un registre sécurisé et chiffré de transactions. Ces transactions étaient réalisées dans une monnaie désormais célèbre, le bitcoin.

Rapidement, les industriels de différents secteurs ont compris l'intérêt de ce registre sécurisé dans leur domaine d'activité. L'industrie de la musique a réalisé qu'elle pouvait rendre compte des ventes de morceaux et suivre leur utilisation. Le secteur de l'énergie a utilisé la blockchain pour conserver un registre fiable des consommations électriques. Enfin, l'industrie de la santé s'est penchée sur la blockchain pour sécuriser le partage d'informations sensibles comme les dossiers médicaux ou les données de traçabilité des produits de santé.

\section{Intérêt de la blockchain en santé}

Afin de comprendre l'intérêt de la technologie blockchain dans le domaine de la santé, il est important de saisir les caractéristiques qui la différencient des outils actuels de partage et de gestion des données. 


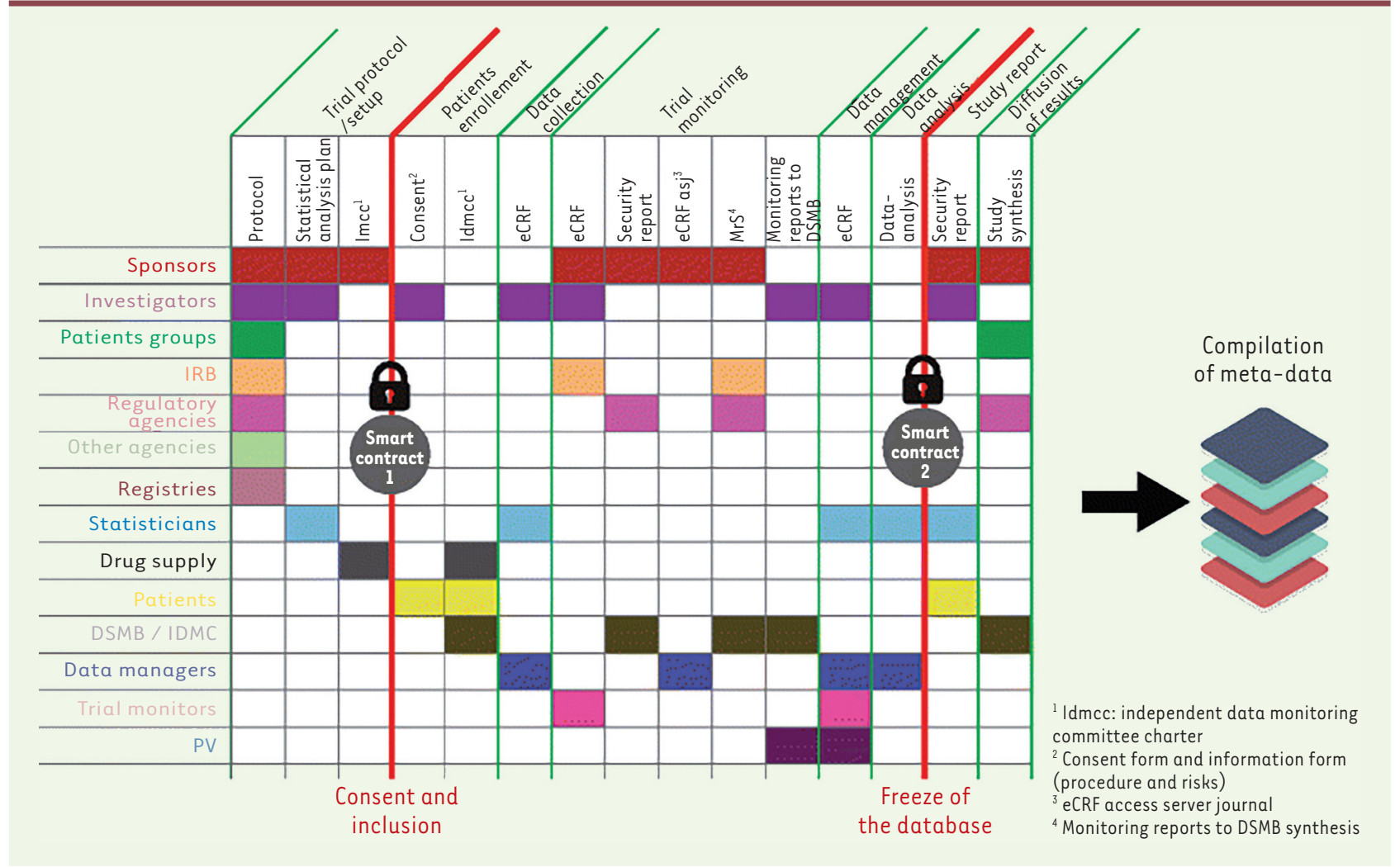

Figure 1. Technologie blockchain pour améliorer la qualité de la recherche clinique. Cette figure indique les sources de données dans un essai clinique ainsi que les parties prenantes qui y ont accès. Deux points sensibles sont identifiés : l'inclusion des patients dépendante du consentement et les rapports fournis suite aux études qui nécessitent que les informations ne soient plus modifiées a posteriori. Ces deux points critiques sont des opportunités d'implémentation de la blockchain (figure issue de [7], sous licence CC BY).

Tout d'abord, la blockchain est une technologie décentralisée. Contrairement aux outils classiques qui sont gérés de manière centralisée par un intermédiaire unique, la blockchain est une base de données distribuée entre tous les nœuds du réseau. Ainsi, chaque mineur dispose d'une copie du registre. Cette technologie se prête ainsi au partage d'informations entre plusieurs parties prenantes comme les hôpitaux, les centres de recherche ou les laboratoires pharmaceutiques [1, 2]. La blockchain permet également de tracer l'origine et le devenir de chacune des données enregistrées. Contrairement à un système centralisé dans lequel un administrateur contrôle ces informations, dans une blockchain, seul le propriétaire peut les enregistrer et les transférer aux autres parties prenantes. De plus, bien que le registre créé par la blockchain soit mis à disposition de tous les membres du réseau, les données qu'il contient sont chiffrées, ce qui permet d'assurer la confidentialité et la sécurité des données sensibles [3].

\section{Applications en santé}

\section{Gestion des données médicales}

De nos jours, il est difficile de visualiser de manière claire toutes les données liées à un patient et accumulées au cours de son parcours de soins. Ces informations proviennent habituellement de sources très variées, comme les médecins de ville, l'hôpital, les assurances, les pharmaciens ou les laboratoires d'analyses médicales. Les logiciels utilisés par ces parties prenantes pour collecter et gérer les dossiers médicaux sont différents et ne sont pas interopérables. Ainsi, il est parfois difficile de partager les informations. Par ailleurs, même si ces informations peuvent toutes être récupérées et assemblées, il n'est pas toujours évident de savoir dans quel ordre elles ont été produites et si elles sont exhaustives. Ce problème est fréquemment rencontré lors de l'admission d'un patient à l'hôpital. Les professionnels de santé n'ont en effet pas toujours accès à son historique et n'ont pas une visibilité complète sur les traitements qu'il prend, sur l'historique de sa maladie ou sur ses antécédents familiaux.

L'idéal serait donc d'avoir une liste qui répertorie tous les lieux où se trouvent les données médicales d'un même patient afin de pouvoir rapidement les récupérer. Cette liste serait accessible, avec l'accord du patient, à tout professionnel de santé qui en ferait la demande. Ainsi, plutôt que de n'avoir accès qu'à la base de données de l'établissement où l'on se trouve, on pourrait 
avoir accès à toutes les sources d'informations dispersées dans toutes les bases de données du réseau. La technologie blockchain apporte justement cette solution sous la forme d'un registre distribué et sécurisé qui permet au patient non seulement d'avoir une visibilité sur ses données, mais aussi d'en contrôler les accès [4].

\section{L'optimisation des essais cliniques}

Les essais cliniques sont une étape obligatoire dans le développement d'un médicament: ils correspondent le plus souvent à la première administration d'un médicament chez l'homme. La participation des patients est donc la condition sine qua non pour que des essais cliniques puissent être réalisés. Le consentement éclairé du patient doit obligatoirement être recueilli avant le début de l'essai. En effet, la déclaration d'Helsinki stipule que chaque sujet doit être informé, entre autres, de l'objectif, des méthodes, des sources de financement et des avantages et des risque anticipés. Par ailleurs, le consentement recueilli pour un protocole ainsi que pour toutes ses révisions doit être transparent et traçable par les patients et les autorités. En pratique, la rigueur fait parfois défaut. Ainsi, la FDA (Food and drug administration) aux États-Unis fait état de ce manquement dans près de $10 \%$ des essais contrôlés : absence de consentement, utilisation de formulaires non approuvés, documents invalides, non-consentement à un protocole révisé.

Par l'inviolabilité et la traçabilité des données, la blockchain permettrait de constituer un socle solide à l'inclusion des patients dans les essais cliniques. En effet, la blockchain peut horodater et stocker les étapes du processus de consentement assurant leur authenticité et leur traçabilité. Cela permettrait aux responsables de la recherche clinique de partager les demandes de consentement en temps réel. Les protocoles de ces essais peuvent également être enregistrés sur la blockchain permettant aux patients de recevoir une notification à chaque changement de protocole, nécessitant de nouveau la collecte du consentement. L'obtention de ce dernier doit être un «verrou » qui empêche la poursuite de l'étude s'il n'est pas obtenu. Les conditions de poursuites de l'essai peuvent être codées dans la blockchain sous la forme de smart contract (contrats intelligents) : «SI (condition) ALORS (conséquence) ». Ce système déclenche automatiquement un événement lorsque les clauses codées sont remplies. Dans le cas d'un formulaire de consentement, le smart contract pourrait indiquer : SI le consentement du patient est enregistré avec sa signature numérique unique, ALORS le patient peut être inclus dans l'essai $[5,6]$.

Un grand nombre de données est généré puis analysé pour démontrer une balance bénéfice/risque positive, autrement dit, une efficacité et une sécurité d'emploi d'une molécule. L'enjeu est donc ici de garantir la reproductibilité de ces essais. Selon loannidis et al. [7], $80 \%$ des essais seraient non reproductibles. De nombreuses divergences apparaissent en effet entre les informations envoyées à l'agence réglementaire et celles présentées dans les publications. S'ajoute à cela, la non publication des résultats d'un grand nombre d'essais cliniques après l'approbation de l'agence, malgré les directives de l'OMS (Organisation mondiale de la santé) en 2015 et de la FDA.
La blockchain apparaît donc comme une solution permettant non seulement de garantir l'intégrité des données et leur traçabilité, mais aussi de préserver la confidentialité des patients. En effet, chaque résultat et document produits pendant l'essai peut être enregistré sur une blockchain privée, gérée par les régulateurs, la société pharmaceutique et les organismes de recherche. L'information devient alors immuable et transparente. De plus, lorsque la confidentialité des données est requise, les informations peuvent être chiffrées, assurant ainsi le fait que les données identifiables concernant le patient ne peuvent pas être dévoilées. La Figure 1 représente les flux complexes d'informations échangés dans un essai clinique et les interactions possibles avec la blockchain [6].

Ainsi, l'avantage principal de cette technologie blockchain est l'augmentation de la transparence et de la fiabilité des protocoles et des résultats des essais cliniques. Cependant, une des conditions essentielles à l'utilisation de cette technologie pour la traçabilité des informations issues d'essais cliniques est la numérisation des données. Or, à l'heure actuelle, la plupart des données sont générées sur une série de documents papier. De plus, on se heurte dans certains pays au cadre réglementaire qui entoure ces essais cliniques. Par exemple, la législation française requiert que le consentement du patient soit donné par écrit. Le consentement numérique enregistrable sur une blockchain serait donc impossible. Aux États-Unis, cependant, la FDA a donné son accord pour la numérisation des processus d'information et de consentement du patient laissant ainsi place à une solution blockchain.

\section{Traçabilité des médicaments et lutte contre la contrefaçon}

Selon I'OMS, près de $15 \%$ de médicaments falsifiés sont en circulation dans le monde. Ce fléau mondial entraîne des conséquences graves voire dramatiques sur la santé des patients. En effet, ils ne répondent à aucune des exigences de qualité, d'efficacité et de sécurité, entraînant ainsi près de 800000 décès chaque année. La falsification peut concerner à la fois les produits de marques et les produits génériques, et touche l'ensemble des aires thérapeutiques [8].

En 2011, le médicament détrônait la contrefaçon de cigarette, le mettant ainsi en tête des produits contrefaits. Ce phénomène, attribué le plus souvent aux pays en voie de développement, n'épargne pas les pays développés comme le montre les différents scandales de ces dernières années. En 2012, des anticancéreux falsifiés ont été découverts dans 19 cabinets médicaux aux États-Unis. Ceci s'explique en partie avec l'essor du 


\section{Blockchain pour la traçabilité des médicaments}

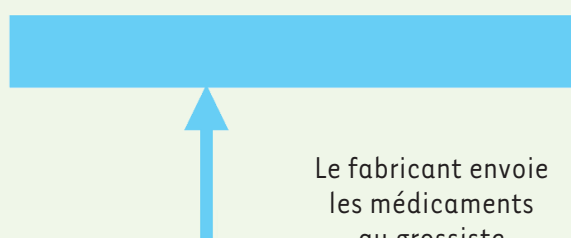

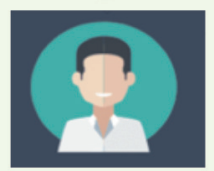

Le fabriquant produit le médicament et le marque avec un code unique

\# Un hash est produit

ถ⿻ au grossiste

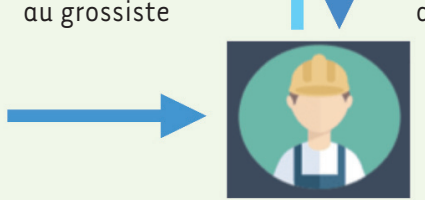

\# Le grossiste vérifie l'origine du produit
Le grossiste envoie les médicaments au pharmacien

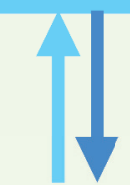

Le pharmacien délivre le médicament au patient

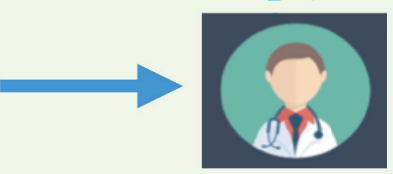

\# Le pharmacien vérifie

$\#$

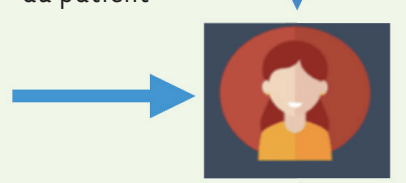

\# Le patient vérifie l'origine du produit

ה) fabriquant et grossiste
est inscrite dans la blockchain
ล) Le transaction entre
grossiste et pharmacien
est inscrite dans la blockchain
ถ) pharmacien et patient
est inscrite dans la
blockchain

Figure 2. Technologie blockchain pour la traçabilité des médicaments. Cette figure retrace le parcours du médicament depuis le fabricant jusqu'au patient. Chaque interaction d'un maillon de la chaîne de distribution avec le produit génère une empreinte numérique (appelée hash) qui est stockée dans la blockchain.

commerce sur internet qui favorise largement la pénétration de médicaments contrefaits en Europe et aux États-Unis. Selon une statistique de I'OMS, la proportion des médicaments contrefaits achetés sur des sites internet illégaux serait d'environ $50 \%$ et représenterait 75 milliards de dollars [9].

Ainsi, parallèlement à l'impact catastrophique sur la santé publique, la contrefaçon engendre des coûts conséquents. Le marché de la contrefaçon de médicaments génère en effet 200 milliards de dollars par an et représente un manque à gagner de 10,2 milliards d'euros pour le secteur pharmaceutique européen.

Malgré les efforts de l'industrie pharmaceutique ainsi que des États pour enrayer ce fléau, la contrefaçon perdure et se propage dans certaines régions de façon exponentielle. L'une des solutions possibles serait d'agir sur le système de traçabilité. Ainsi la blockchain, de par son niveau de sécurité, apparaît comme une technologie révolutionnaire dans la traçabilité des médicaments. En effet, l'idéal serait d'enregistrer chaque étape de la chaîne de distribution d'un médicament et de combiner ainsi l'usage de la blockchain à celui des différentes technologies de traçabilité telles que les puces RFID (radio frequency identification) ou les code-barres Datamatrix ${ }^{1}$. Chaque nouvelle transaction ajoutée à un bloc est ainsi immuable et horoda-

${ }^{1}$ Code-barres bidimensionnels à haute densité. tée, ce qui permet de suivre la chaîne du médicament et de s'assurer que les informations le concernant ne sont pas altérées. L'ensemble des parties prenantes (acteurs de la chaîne [supply chain], patients) pourraient avoir accès à ce système et vérifier la provenance et l'intégrité des médicaments. Par ailleurs, selon la position de chacun dans la chaîne de distribution, les droits seront différents : les laboratoires peuvent enregistrer de nouveaux médicaments alors que les grossistes ne peuvent que valider le passage du produit permettant ainsi une maîtrise et une sécurité accrue de la chaîne (Figure 2). La technologie blockchain apporte donc deux réels avantages en termes de traçabilité des médicaments: elle permet un suivi des produits dans la chaîne de distribution, créant un circuit hermétique, imperméable aux produits contrefaits; elle permet également aux parties prenantes, et en particulier aux laboratoires, de prendre des mesures a posteriori, en cas de problème, en identifiant l'emplacement exact de leurs médicaments.

Cependant, l'implémentation de cette technologie pour ce cas d'usage nécessite une chaîne de distribution fonctionnelle dans laquelle l'ensemble des acteurs 
sont identifiés. Dans les pays industrialisés tels que la France ou les États-Unis, celle-ci est globalement maitrisée. Dans les pays en voie de développement, ces chaînes sont parfois défectueuses et l'identification claire de l'ensemble des intermédiaires reste impossible. Ainsi, utiliser une blockchain dans ces conditions n'apporterait pas de réel avantage puisqu'il faudrait au préalable déterminer les maillons de la chaîne afin d'implémenter le système auprès de chacun d'entre eux.

\section{Conclusion}

En conclusion, cette liste non exhaustive des applications potentielles de la blockchain en santé montre l'impact réel sur l'organisation et l'efficience du système de soins. Cependant, il est important de noter que cette technologie n'est qu'un outil et non une solution à tous les maux de l'industrie de la santé. L'implémentation de la blockchain ne sera possible qu'à la suite d'une réelle coopération de l'ensemble des parties prenantes. Il est donc nécessaire de réaliser un travail d'amont important de numérisation des données, d'automatisation des processus, d'éducation du personnel et d'encadrement réglementaire. $\diamond$

\section{SUMMARY}

Opportunities and challenges of blockchain technology in the healtheare industry

Often described as a tool to build trust among stakeholders with divergent interests, blockchain technology has been of interest to many sectors since it was first used in 2008. Initially designed to record financial transactions between individuals, its applications have largely evolved with technological advances and the growing interest of international companies. In the healthcare sector, blockchain is interesting for many of its features: its immutability which makes it an excellent support for authenticating sensitive data such as clinical trials consents, the possibility of publishing smart contracts that automate and facilitate many processes or the constitution of a net- work that agrees on the state of the information. Much acclaimed, blockchain technology is still to be tested in real-life conditions and adapted to a particularly complex regulatory and economic context in the healthcare sector. $\diamond$

\section{LIENS D'INTÉRÊT}

Les auteurs déclarent n'avoir aucun lien d'intérêt concernant les données pubiées dans cet article.

\section{RÉFÉRENCES}

1. Till BM, Peters AW, Afshar S, Meara J. From blockchain technology to global health equity: can cryptocurrencies finance universal health coverage? BMJ Global Health 2017 ; 2 : e000570.

2. Angraal S, Krumholz HM, Schulz WL. Blockchain technology: applications in health care. Circ Cardiovasc Qual Outcomes 2017 ; 10 : e003800.

3. Gupta V. A brief history of blockchain. Harvard Business Review. 28 février 2017. https://hbr.org/2017/02/a-brief-history-of-blockchain

4. Kuo TT, Kim HE, Ohno-Machado L Blockchain distributed ledger technologies for biomedical and health care applications. J Am Med Inform Assoc 2017 ; 24 : 1211-20,

5. Benchoufi M, Ravaud P. Blockchain technology for improving clinical research quality. Trials $2017 ; 18: 335$.

6. Benchoufi M, Porcher R, Ravaud P. Blockchain protocols in clinical trials: transparency and traceability of consent. F1000Research $2017 ; 6: 66$.

7. loannidis JPA. Why most published research findings are false. PLoS Med $2005 ; 2$ : el24.

8. Rapport du LEદM. Contrefaçon de médicaments, une atteinte à la santé publique. Paris : Leem, juin 2017. http://www.leem.org/sites/default/files/ DP-contrefacon-06-07-2017.pdf]

9. Organisation Mondiale de la Santé. L'OMS et ses partenaires accélèrent la lutte contre les médicaments contrefaits. Genève : 0MS, 2006. http://www. who.int/mediacentre/news/releases/2006/pr69/fr/]

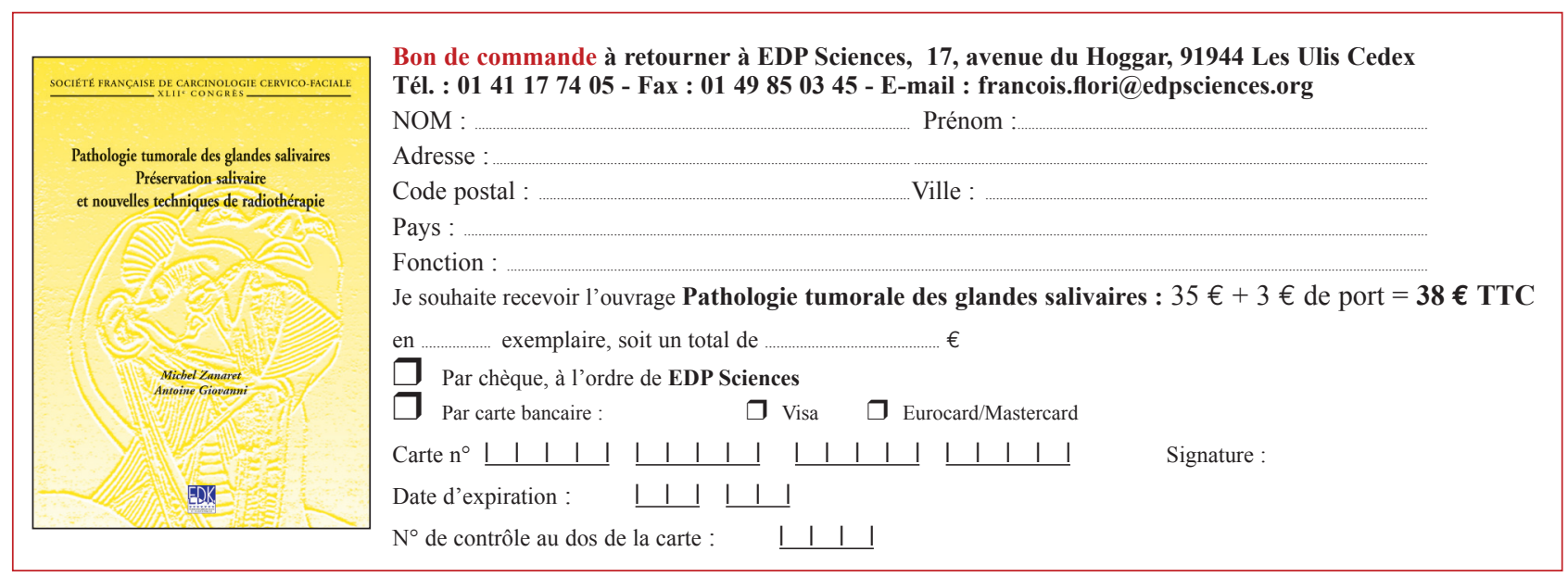

TIRÉS À PART

A. Petre 